\title{
The Application of Mathematical Morphology in the Disturbance Detection of Power Quality
}

\author{
Yanqin $\mathrm{Hu}^{1}$ and Yin $\mathrm{Gao}^{2}$ \\ ${ }^{1}$ State Gird Jiangxi Electric Power Research Institute; \\ ${ }^{2}$ Nanchang Hangkong University
}

Keywords: Mathematical morphology; Structural elements; Power quality; Detect location.

\begin{abstract}
A method combining $\alpha \beta$ conversion and $\mathrm{d} \mathrm{q}$ conversion to detect power quality disturbances is proposed based on previous studies, which makes use of the characteristics of voltage perturbation signal. It designs two filter elements by choosing a suitable structural element to locate the voltage perturbation signal. The simulation results show that the proposed method is simple, and they can locate the disturbances of the voltage signals fast, accurate
\end{abstract}

\section{Introduction}

Power quality description is the quality of the user side of the power supply through the public power grid [1]. The ideal state of the utility grid with constant frequency sinusoidal and standard voltage to supply power to the users, in a three-phase system, also called amplitude of voltage and current in each phase are equal in size and symmetry phase and should be mutual difference of $120^{0}$.

Electric power is a kind of economic, practical, clean and easy to control and conversion of energy from, its application level is one of the main symbols of a country's level of development. With the development of science and technology, the power system with electric load change and nonlinear load using the waveform distortion, power quality decline, with based on microprocessor of the controller and the power electronic devices modern with the use of electrical equipment, but also improve the requirements on the quality of electric energy. In addition, the concept of information power called for people to pay attention to the dynamic power quality issues on the information society of the serious harm [2]. Therefore, the study of power quality has become a hot issue.

Most of the power quality problems in power system are harmonic pollution and power quality disturbance [3]. Power quality disturbances include voltage sag, voltage sag, voltage interruption and transient oscillation. The United Kingdom in 1995 on the power quality problem to do a survey, 100 of the user capacity of more than 1MW. The results show that in the monitoring period of 12 months, $69 \%$ of the users in the production process is affected by the power quality problems, of which $83 \%$ of the accidents are caused by voltage sag and interruption caused [4].

\section{Concept of Mathematical Morphology}

Mathematical morphology [5] is a mathematical analysis method based on strict mathematical theory, such as integral geometry and random set theory, which has the advantages of simple calculation and parallel computation.

The basic operation of mathematical morphology, including expansion, corrosion, and thus leads to the open and close operation, the power sampling signal is generally one-dimensional data, so only the gray value image algebra mathematical morphology method is introduced.

The signal $\mathrm{f}(\mathrm{n})$ is a one dimensional multi valued signal obtained by sampling, and its definition domain is $\mathrm{D}[\mathrm{f}]=\{0,1,2,3, \ldots, \mathrm{N}\} ; \mathrm{G}(\mathrm{x})$ is a sequence of one dimensional structure, its definition domain is $\mathrm{D}[\mathrm{g}]=\{0,1,2,3, \ldots, \mathrm{P}\}$; The $\mathrm{P}$ and $\mathrm{N}$ is an integer, then the corrosion and expansion operations were defined as follows:

$$
(f \Theta g)(n)=\min \{f(n+x)-g(x): x \in D[g]\}
$$




$$
\begin{aligned}
& (n=1,2,3, \cdots N) \\
& \quad(f \oplus g)(n)=\max \{f(n-x)+g(x): x \in D[g]\}
\end{aligned}
$$

$(n=1,2,3, \cdots N)$

In the formula, $\Theta$ represents the corrosion operation. (Indicates the expansion operation.

The definitions of morphological open operation and close operation are as follows:

$(f \circ g)(n)=(f \Theta g \oplus g)(n)$

$(f \bullet g)(n)=(f \oplus g \Theta g)(\mathrm{n})$

In the formula, represents an open operation, $\bullet$ represents a closed operation.

The morphological opening and closing operation can be constructed 3 kinds of filtering algorithm based on a hybrid filter, filter; alternate, alternating hybrid filter.

The structural elements of the same width as (the length of structural elements of $\mathrm{P}$ ), the calculation speed alternating filter and hybrid filter similar. Alternate hybrids filter speed, when the $\mathrm{P}$ value is large, the time difference will be greater. The output of the filter will usually be alternate down (or up). This is because open (closed) output signal processing is always located in the original signal (on) the reason. In order to solve the problem of signal output, a mixed filter or an alternative hybrid filters. This chapter uses the alternate hybrid filter.

Alternating hybrid filter:

$[(f) \operatorname{ah}(g)](n)=[(f) \operatorname{oc}(g)+(f) \operatorname{co}(g)](n) / 2$

Among them, OC showed that the first open and then closed alternating filter operation; $\mathrm{CO}$ showed that the first closed after the alternating filtering operation; her representation of hybrid filtering operation; ah representation of alternating mixed filtering operation.

\section{The Principle of Disturbance Localization and Its Algorithm Construction}

Disturbance Detection and location. For single-phase voltage disturbance, if by constructing a virtual symmetrical three-phase system to detect the disturbance occurred in the moment, it is difficult to carry out accurate positioning. According to the $\alpha \beta$ and $\mathrm{dl}$ transform. As showed in the formula (6), the $u \alpha$ and $u \beta$ components can be constructed directly from a single phase voltage.

$$
\begin{aligned}
& {\left[\begin{array}{l}
u_{d} \\
u_{q}
\end{array}\right]=} \\
& \sqrt{\frac{2}{3}}\left[\begin{array}{cc}
\sin w t & -\cos w t \\
-\cos w t & -\sin w t
\end{array}\right] \cdot\left[\begin{array}{ccc}
1 & -1 / 2 & -1 / 2 \\
0 & \sqrt{3} / 2 & -\sqrt{3} / 2
\end{array}\right]\left[\begin{array}{l}
u_{a} \\
u_{b} \\
u_{c}
\end{array}\right]
\end{aligned}
$$

Without loss of generality, a voltage signal containing disturbance as shown in (7), $N(t)$ for disturbance signal

$$
u_{1}=V_{m 1} \sin (w t+\alpha)+N(t)
$$

$\mathrm{u} 1$ delays will be a small angle $\theta$ ( $\Delta \mathrm{t}$ is relative to the $\theta$ should be the time delay), can be got:

$u_{2}=V_{m 1} \sin (w t+\alpha-\theta)+N(t-\Delta t)$

Marks

$$
\begin{aligned}
& u_{3}=u_{2} \cos \theta-u_{1}=-\sin \theta V_{m 1} \sin (w t+\alpha-\theta) \\
& +N(t-\Delta t) \cos \theta-N(t)
\end{aligned}
$$


$u_{\alpha}=u_{2}=V_{m 1} \sin (w t+\alpha-\theta)+N(t-\Delta t)$

$u_{\beta}=(1 / \sin \theta) u_{3}=-V_{m 1} \cos (w t+\alpha-\theta)$

$+N(t-\Delta t) c \tan \theta-N(t) / \sin \theta$

$D l$ transformation of $u_{\alpha}$ and $u_{\beta}$,

$$
\begin{aligned}
& {\left[\begin{array}{l}
u_{d} \\
u_{q}
\end{array}\right]=\left[\begin{array}{cc}
\sin w t & -\cos w t \\
-\cos w t & -\sin w t
\end{array}\right]\left[\begin{array}{l}
u_{\alpha} \\
u_{\beta}
\end{array}\right]} \\
& =\left[\begin{array}{c}
V_{m 1} \cos (\alpha-\theta)+H_{1} \\
-V_{m 1} \sin (\alpha-\theta)+H_{2}
\end{array}\right]
\end{aligned}
$$

Among them,

$$
\begin{aligned}
& H_{1}=1 / \sin \theta \cdot \cos w t \cdot N(t) \\
& +(\sin w t-c \tan \theta \cos w t) \cdot N(t-\Delta t) \\
& H_{2}=1 / \sin \theta \cdot \sin w t \cdot N(t) \\
& +(\cos w t+c \tan \theta \sin w t) \cdot N(t-\Delta t)
\end{aligned}
$$

By the formula (12) shows that $\mathrm{X}$ and $\mathrm{Y}$ are a DC component, and satisfies the formula (15):

$$
\left[V_{m 1} \cos (\alpha-\theta)\right]^{2}+\left[-V_{m 1} \sin (\alpha-\theta)\right]^{2}=V_{m 1}^{2}
$$

Power quality disturbances can be considered to be loaded in the ideal power system communication $50 \mathrm{~Hz}$ sine wave non-stationary signal; when the system does not exist, the morphological filter is very good to weaken the noise and does not change the performance of the low frequency signal. Therefore, demising using morphological filter, the type (12) in H1 and H2 are close to zero, then the XX and TTT square and can get approximate detection voltage amplitude square value U2. According to the change in the study period the voltage amplitude square can detect amplitude disturbance signal of the time and the disturbance signal, so as to realize the detection of disturbance location on voltage signal.

Algorithm Construction. According to the characteristics of mathematical morphology and the detection principle of disturbance, we can know that the realization of the disturbance detection algorithm needs to pay attention to several aspects: (1) select the appropriate structural elements to establish the morphological filter;

(2) Because the morphological filter is based on the basic operations of mathematical morphology, and the signal will occur in the operation process of the expansion and corrosion loss, so the disturbance signal denoising should guarantee the authenticity of the signal with morphological filter.

(3) Set the appropriate morphological filter series, as showed in Fig. (12). At this time for the $u_{d}$ and $u_{q}$ components should be morphological filtering.

Algorithm flow of power quality disturbance detection and location are shown in Fig. 1.

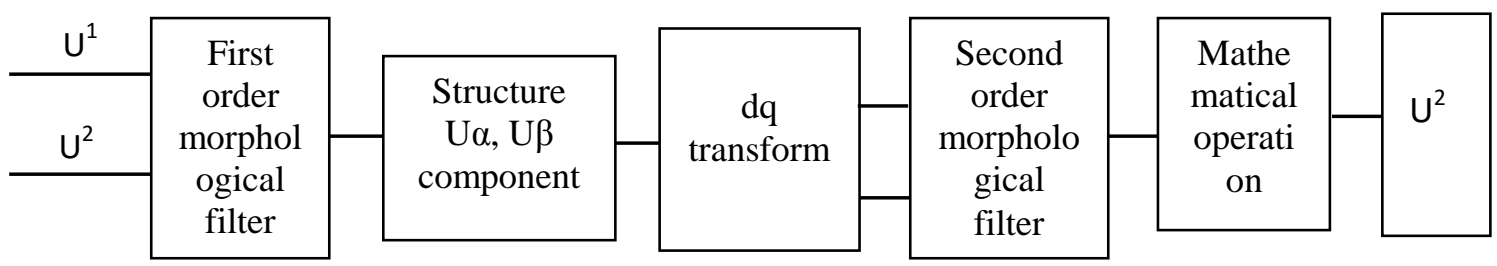

Figure 1. The mathematical form of power quality disturbance detection based on block diagram 
The delay signal $u_{2}$ the value of $\theta$ in the smaller, faster dynamic response, but the $\theta$ value is too small, the noise in the system will have great influence on the baseband signal. Through a lot of simulation, the selection of $\theta$ value in the $10^{\circ}$ has a better effect ${ }^{[6]}$, the simulation example is selected $\theta=7.2^{\circ}, \Delta t=0.0004 \mathrm{~s}$. The first order morphological filter in length is selected for the five sampling points and sampling frequency of $10 \mathrm{kHz}$ ), semi-circle of radius $r=0.1$ as structural elements, $\mathrm{G}(\mathrm{n})=\{0,0.3442,0.1,0.3442$. $\}$ And filter algorithm adopts the alternating mixed filter algorithm, such as (5) the show; the second order morphological filter to select length of three sample point of linear structures, filtering algorithm is still in alternative hybrid filtering algorithm.

Disturbance detection block diagram, the power quality disturbance signal $u 2$ and the disturbance delay signal $\mathrm{u} 1$, first through the first stage filter to eliminate noise. Then, after denoising the signal obtained by constructing $\mathrm{u}$ alpha and $\mathrm{u}$ beta component, and dq transform $\mathrm{ud}$ and uq components, according to the detection principle of disturbance location set of second filters, filter $\mathrm{H} 1$ and $\mathrm{H} 2$ components, and finally get the voltage situation during the period of study by mathematical operations.

\section{Simulation Example}

Voltage Drop. In the study period, according to the disturbance detection block diagram based on the mathematical morphology, the mathematical form of the disturbance detection block diagram is analyzed, and the waveform diagram of the variation of each component of the whole disturbance detection block diagram is shown in Fig. 2 to 10.

Fig. 2 shows for the system disturbance caused by voltage sag waveform. Among them, design voltage drops to 0.5 (Standard unitary value), drop from ending time respectively $0.035 \mathrm{~s}$ and $0.075 \mathrm{~s}$ that drop from check of sampling points respectively in 350 and $750 \mathrm{~nm}$, Fig. 3 is after the first morphological filter denoting after the waveform. At this point, the need to set up second morphological filter to detect voltage disturbance, Fig. 4 shows the u beta component of the waveform. Fig. 5 and Fig. 6 are the ud and uq component waveforms after dq transform, from which the amplitude variation of the disturbance signal can be roughly detected in Fig. 5, and the starting and ending time of the disturbance signal can be located in Fig. 6. At this time of ud, uq component using second order morphological filtering algorithm further elimination noise, from Fig. 7 known study period voltage amplitude of fluctuations, in sampling of voltage amplitude in $350 \sim 750$ for 0.5 , and in the other voltage amplitude for standard unitary value 1. From Fig. 8 the voltage disturbance occurred time and voltage amplitude disturbance changes direction, namely small change or from small to large. Shown in Fig. 8, the sampling point 350 voltage amplitude changes from big to small, the sampling point 750 voltage amplitude changes from small to large. Fig. 9 shows the fluctuation of voltage amplitude squared after mathematical operation.

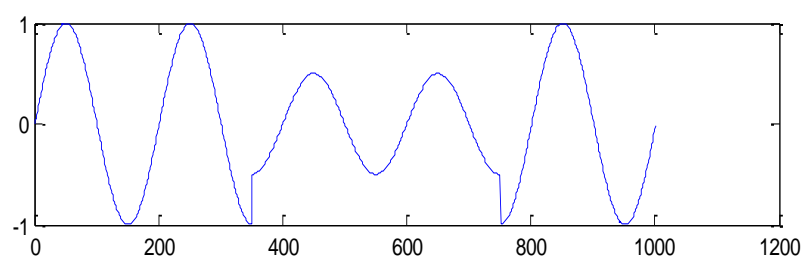

Figure 2. Voltage sag waveform caused by system disturbance $u_{1}$

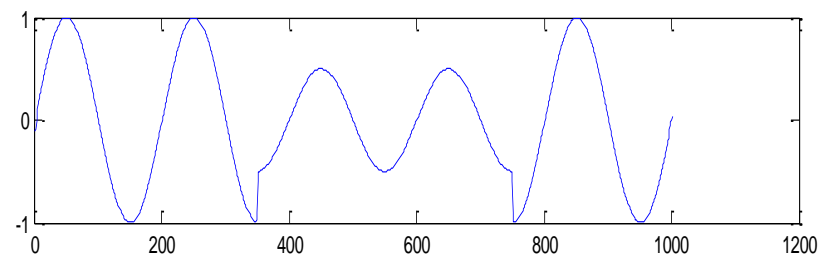

Figure 3. After the first morphological filtering algorithm $u_{1} 、 u_{2}\left(u_{\alpha}\right)$ 


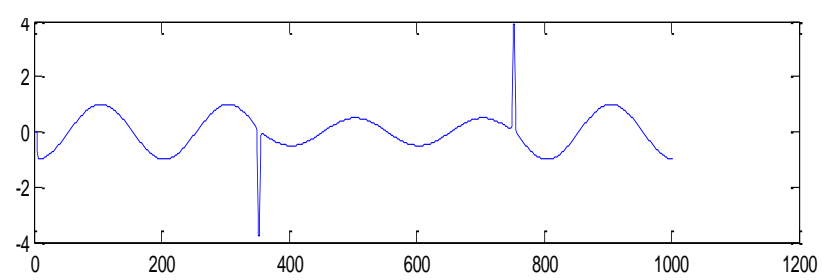

Figure $4 . \quad \mathrm{u}_{\beta}$ component

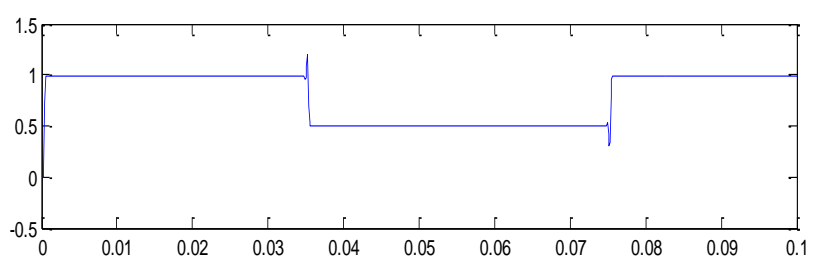

Figure 5. $u_{d}$ component

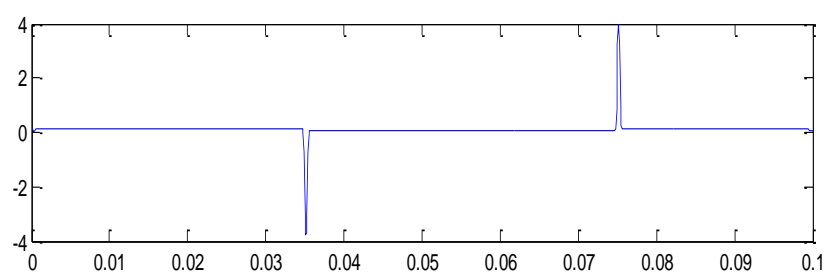

Figure 6. $u_{q}$ component

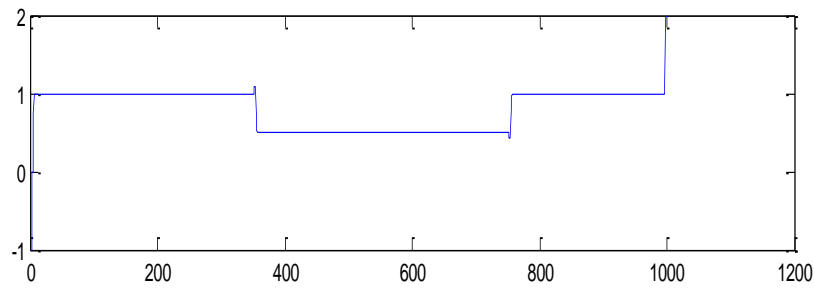

Figure 7. $u_{d}$ component after second stage filtering algorithm

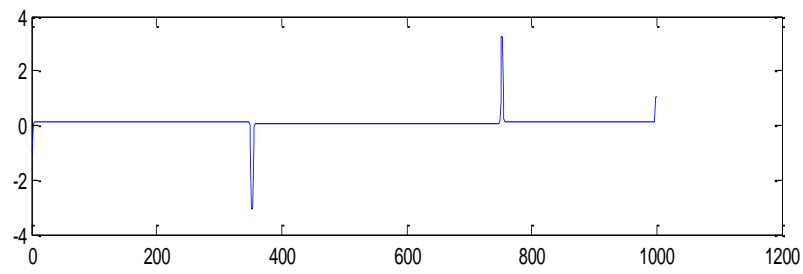

Figure 8. Qu component after second stage filtering algorithm

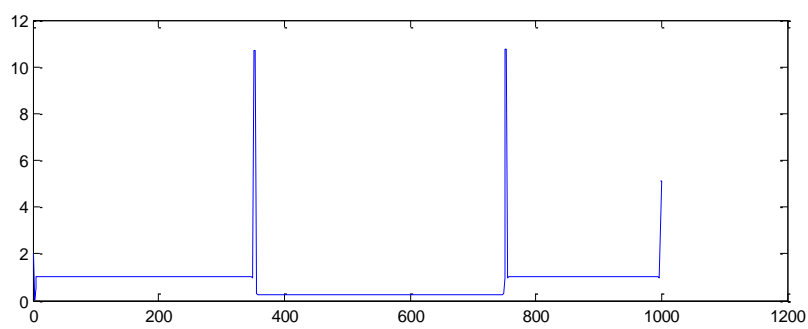

Figure 9. The approximate value of the square of the voltage magnitude U2 


\section{Voltage Swell}

In this paper, the voltage of the system is set up in the normal operation condition. As shown in Fig. 1 based on mathematical morphology disturbance detection diagram analysis, Fig. 10 shows for the system suffered system caused by the voltage swell waveforms, which sets the voltage disturbance amplitude is 1.5 (Standard unitary value) disturbance occurred in a period $0.035 \mathrm{~s} \sim 0.075 \mathrm{~s}$ corresponding disturbance of sampling points for 350 750. Fig. 11 and 12 after two level morphology filtering algorithm after the $u_{d}, u_{q}$ component, the perturbation situation of the voltage amplitude in Fig. 11 shows 0 0.1s. In sampling points at 350 750 voltage amplitude at for 1.5, from Fig. 12 shows the voltage fluctuation stop time is about 0.0353 0.075s. Fig. 13 shows the fluctuation of the amplitude of the disturbance voltage during the study period.

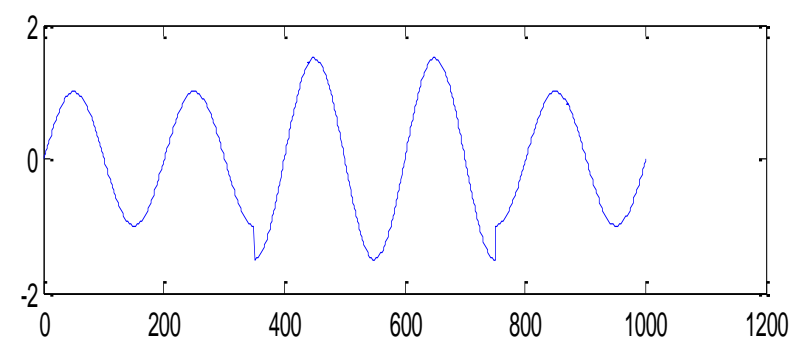

Figure 10. Voltage sag caused by system disturbance u1

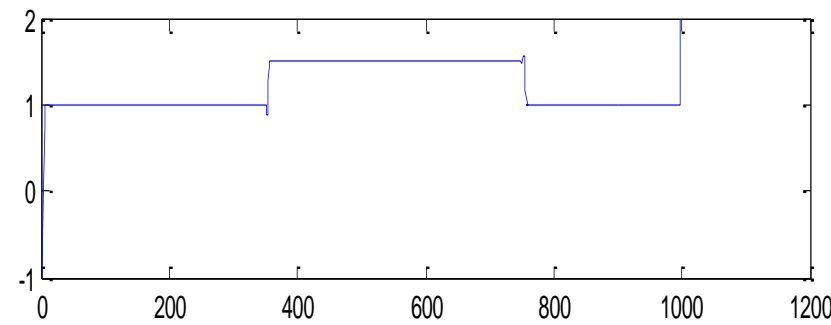

Figure 11. ud component after second stage filtering algorithm

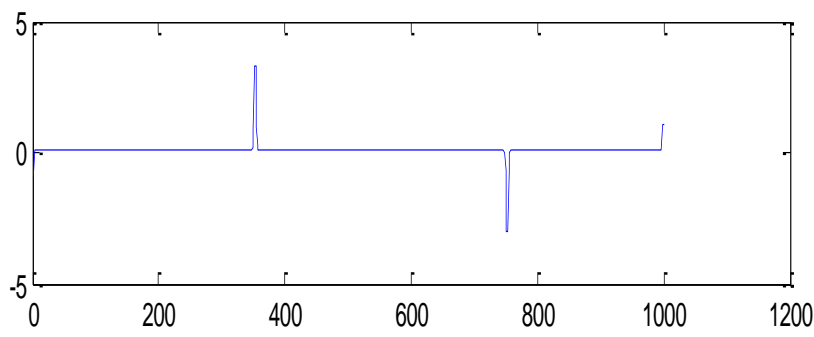

Figure 12. uq component after second stage filtering algorithm

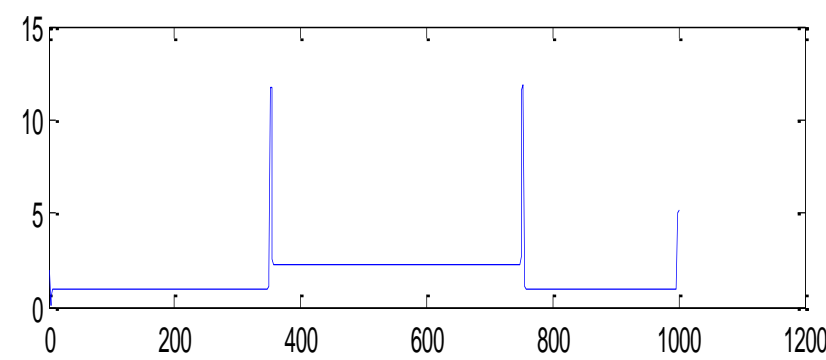

Figure 13. The approximate value of the square of the voltage amplitude U2

\section{Voltage Interruption}

Fig. 14 to Fig. 21 shows the waveform of each component in the block diagram. In Fig. 19, we can know the disturbance of voltage amplitude during the study period. At the sampling point 350 
voltage interruption occurs, the voltage magnitude is reduced to 0 , and the voltage at the sampling point 650 is recovered to the standard value of 1 . Fig. 20 shows that during the study period voltage disturbance voltage amplitude changes direction, in sampling points of 350 voltage amplitude changes directions from big to small, in the direction of sampling 650 voltage amplitude changes is ascending. Fig. 21 shows the disturbance of voltage amplitude squared in the study period.

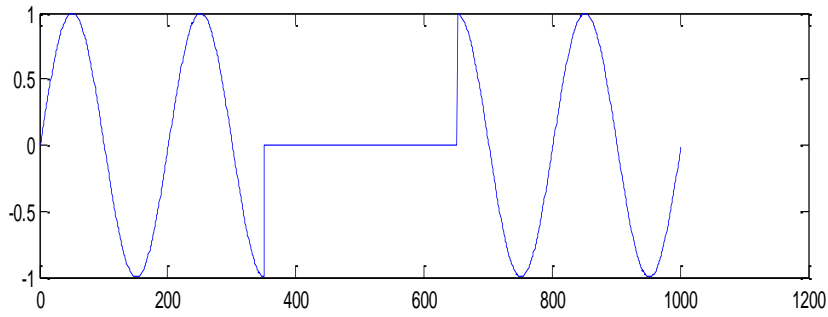

Figure 14. Voltage interruption waveform caused by system disturbance $\mathrm{u} 1$ $(0.035-0.065 \mathrm{~s})$

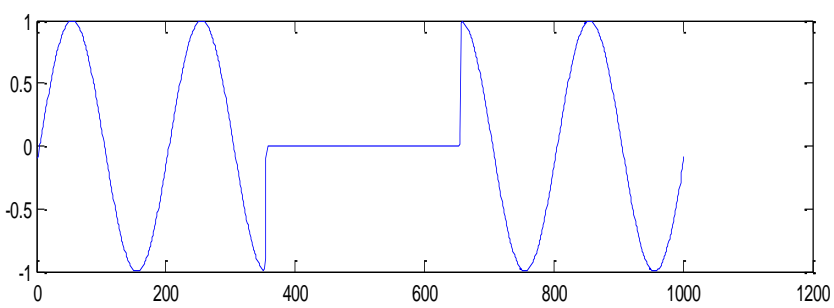

Figure 15. After the first morphological filtering algorithm after $u_{1} 、 u_{2}$ ( $u_{\alpha}$ component)

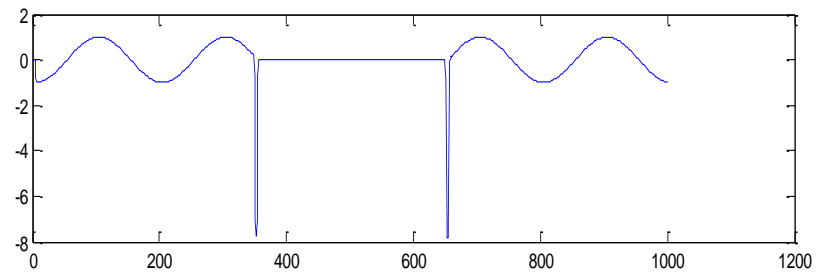

Figure 16. $\mathrm{U} \beta$ component

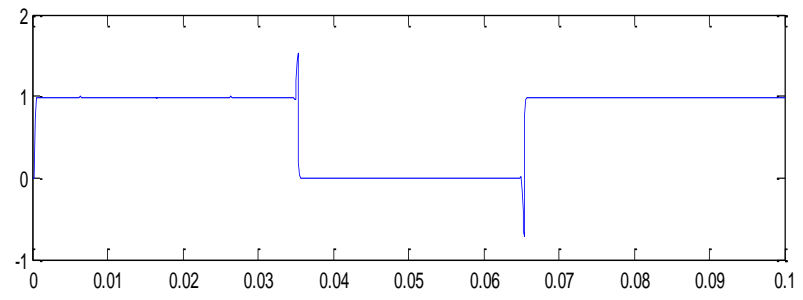

Figure 17. Up component

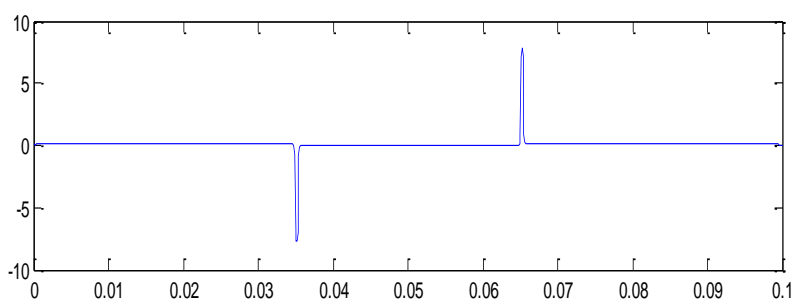

Figure 18. Qu component 


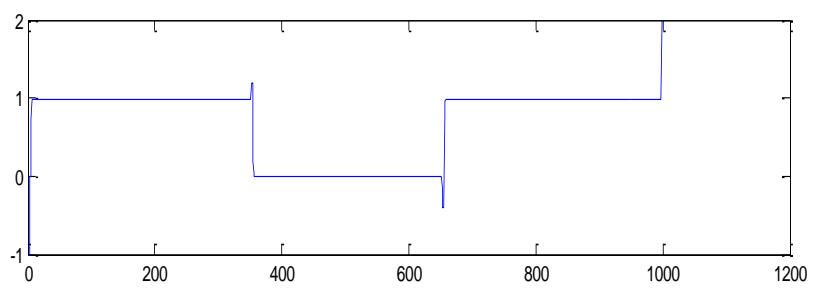

Figure 19. ud component after second stage filtering algorithm

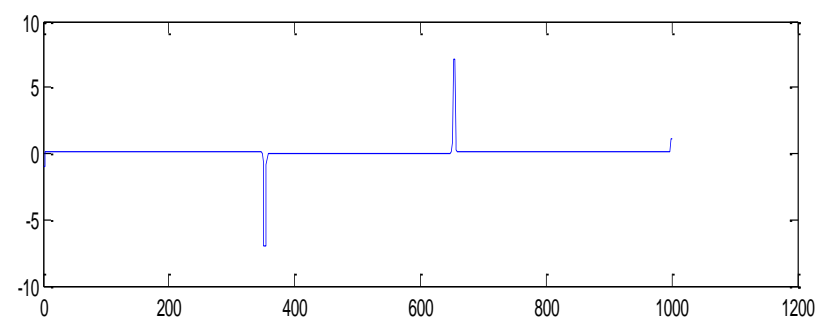

Figure 20. Qu component after second stage filtering algorithm

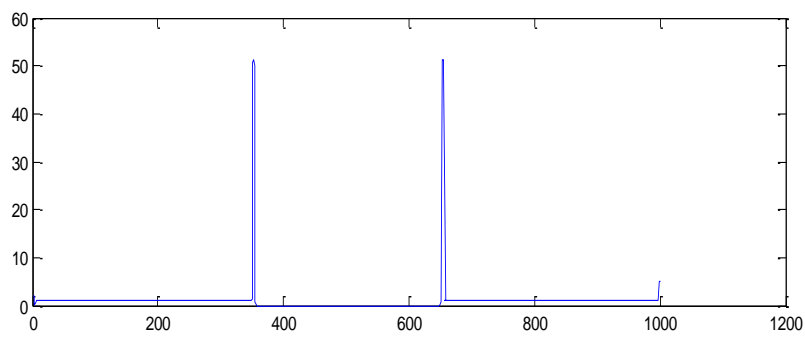

Figure 21. The approximate value of the square of the voltage amplitude U2

\section{Conclusion}

In this paper, on the basis of previous studies, combined with the characteristics of mathematical morphology in image processing of power quality disturbance is studied. In this paper, the power quality disturbance signal is processed by using the $\alpha \beta$ transform and dq transform, and the appropriate structure elements are selected and the 2 stage alternating hybrid filter is adopted to eliminate the noise. Example analysis shows that the method is effective, fast and accurate detection of the disturbance of the voltage.

\section{References}

[1] Cheng Haozhong, Ai Qian, Zhang Zhigang etc. Power Qualit [M]. Beijing: Tsinghua University Press, 2006.

[2] Yan Gangui, Huang Yafeng, Jiang Qirong. Future user power technology [J]. Automation of power station equipment, 2006(01)1-8.

[3] Elmitwally, Kandi M S, Elkateb M. A fuzzy-controlled versatile system for hormones, unbalance and voltage sag compensation[C]. Proceedings of IEEE PES Summer Meeting 2000, Seattle, WA, USA, 2000, 3:1439-1444.

[4] P. L. Clarkson, W. R. Hodgkin. West served customers [UK power quality], Power Engineering Journal, Vol.15, No.2, 2001: 95-99.

[5] Tang Changqing, Lv Hongbo, Huang Zheng etc. Mathematical morphology method and its application [M]. Beijing: Science Press 1990 
[6] Zeng Jiyong, Ding Hongfa, Duan Xianzhong. Harmonic detection and power quality disturbance location method based on mathematical morphology[J]. Chinese Journal of Electrical Engineering, 2005,25(21): 57-62. 